\title{
A MECHATRONICS APPROACH TO THE SERVO DESIGN FOR A MEGLEV SYSTEM
}

\author{
Jia-Yush Yen \\ Professor
}

\author{
Jia-Hua Lin \\ Graduate Student
}

\author{
Department of Mechanical Engineering, \\ National Taiwan University, \\ Taipei, Taiwan 10617, R.O.C. \\ Fax/Vocal: +886-2-2366-0734 \\ e-mail: jyen@ccms.ntu.edu.tw
}

Keywords: Mechatronics; Maglev suspension system; Active stiffness

\begin{abstract}
Magnetic levitation systems have recently become the focus of many research interests because of their frictionless and active stiffness characteristics. However, the maglev systems also represent a high nonlinear and difficult challenge to the control engineers. In this paper, a mechatronics approach is used to simplify the servo design for the maglev systems. By breaking up the system into small SISO loops, enables the use of simple control algorithm to achieve complicated control. An investigation on how to adequately partition the experimental maglev is presented. The analysis enables reasonable subsystem partitions, and achieves overall system behavior that is consistent with the predicted subsystem performance.
\end{abstract}

\section{Introduction}

The maglev (Magnetic Levitation) systems offer many advantages such as frictionless, active stiffness, and the ability to operate in high vacuum environments. These characteristics made the maglev systems particularly attractive to the precision engineering applications [1] [8].

The active stiffness characteristic of the maglev systems arises from the fact that maglev system is inherently unstable and feedback control is necessary to achieve stable operation. Because the system is highly nonlinear, simple classical controllers usually would not work. The tuning process for the time-based controller is very difficult. Many researches have applied very sophisticated control strategies to this problem. Because the maglev systems can vary in different configurations, the controller designs also have to vary accordingly [9] [12]. As a result, these controllers are usually dependent on very precise mathematical models [13]. Maglev systems always have several stabilizing magnets to maintain attitude, therefore nonlinear controls are often necessary. Some of the previous attempts have successfully applied feedback linearization in conjunction with the model based linear-quadratic control [12], or some form of adaptation together with sliding mode control [13] to achieve the desired performance.

This research would like to use a mechatronics approach to reduce the effort involved in the controller design. By decomposing the system into approximate single-input single-output (SISO) linear subsystems, one can apply classical linear controllers to the subsystems. However, an experimental implementation reveals that an intuitive assignment of stable input-output pairs does not guarantee overall system stability. Instead, the coupling effects among the actuators completely messes-up the system behavior. With the help of the kinematics analysis, it is possible to come up with a subsystem division that minimizes the coupling effects. This new decomposition enables a straightforward tuning process. The experimental results are also in accordance with the design goal.

The following section will provide a brief description of the experimental maglev system. The system is designed to achieve long distance and high precision positioning. Section 3 will describe the mechatronics servo design concept and the decomposition of the subsystems. Section 
4 will present the experiment results and compare the different subsystem decomposition. Section 5 will provide a concluding remarks and some further research works.

\section{Repulsive Maglev System Description}

Figure 1 shows the experimental maglev suspension system.

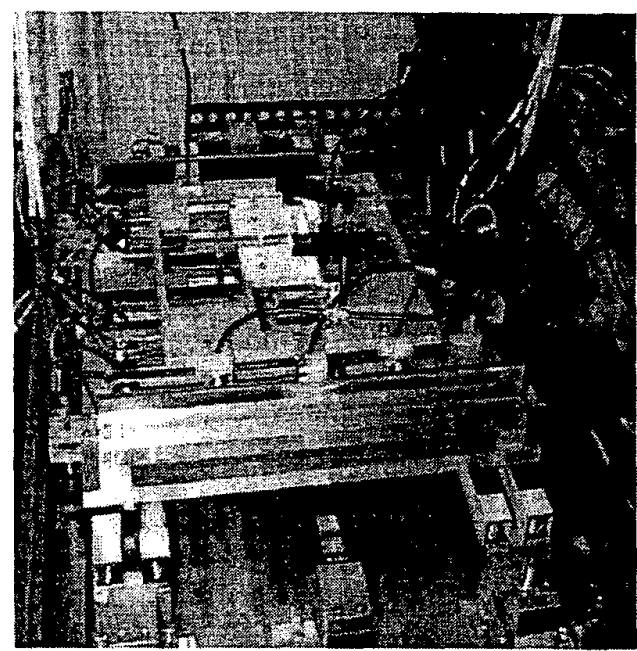

Figure 1. Experimental magnetic levitation stage

Repulsive forces generated by the interaction between two groups of permanent magnets provide the essential lift force. These repulsive levitation forces cause the carriage to be laterally unstable. Separate devices called stabilizers are designed to stabilize this unstable carriage dynamics. The stabilizers apply electromagnetic forces to the carriage magnets used for levitation. The carriage magnets, track magnets and stabilizers constitute the levitation tracks and there are four levitation tracks in the system. Figure 2 shows the six degrees of freedom in the carriage dynamics, $X, Y, Z, \theta, \phi$ and $\psi$, among which the $\theta$ and $X$ degrees of freedom are unstable.

The levitation and literal stabilizing coil generate the necessary control force to maintain the stage attitude. Accordingly, it is necessary to discuss the mechanical dynamics and the electrical dynamics.

\subsection{Mechanical dynamics}

The dynamics of this system can be found in [8], and Huang and Yen derived a more complete nonlinear model in a control-affine form [13]:

$$
\begin{aligned}
& \ddot{x}_{1}=f_{1}\left(x_{1}, x_{2}\right)+g_{11}\left(x_{1}, x_{2}\right) I_{1}+g_{12}\left(x_{1}, x_{2}\right) I_{2}, \\
& \ddot{x}_{2}=f_{2}\left(x_{1}, x_{2}\right)+g_{21}\left(x_{1}, x_{2}\right) I_{1}+g_{22}\left(x_{1}, x_{2}\right) I_{2}
\end{aligned}
$$

where $x_{1}$ and $x_{2}$ are the system dynamic variables $\theta$ and $X$. $I_{1}$ and $I_{2}$ are the current flows in the coil of the electromagnets (stabilizers). $f_{1}, f_{2}, g_{11}, g_{12}, g_{21}, g_{22}$ are nonlinear scalar functions of the form:

$$
\begin{aligned}
f_{1}\left(x_{1}, x_{2}\right)= & \frac{I_{\mathrm{M}} m_{\mathrm{Z}}}{J}\left[2\left(-a_{1}+b_{1} x_{1}\right) \xi_{1}\left(x_{1}, x_{2}\right),\right. \\
& \left.+2\left(a_{2}+b_{2} x_{1}\right) \xi_{2}\left(x_{1}, x_{2}\right)\right] \\
f_{2}\left(x_{1}, x_{2}\right)= & \frac{I_{\mathrm{M}} m_{\mathrm{Z}}}{M}\left[2 \xi_{1}\left(x_{1}, x_{2}\right)+2 \xi_{2}\left(x_{1}, x_{2}\right)\right], \\
g_{21}\left(x_{1}, x_{2}\right)= & \frac{m_{\mathrm{Z}}}{J}\left[2\left(-a_{1}+b_{1} x_{1}\right) \xi_{3}\left(x_{1}, x_{2}\right)\right], \\
g_{12}\left(x_{1}, x_{2}\right)= & \frac{m_{\mathrm{Z}}}{J}\left[2\left(a_{2}+b_{2} x_{1}\right) \xi_{4}\left(x_{1}, x_{2}\right)\right], \\
g_{21}\left(x_{1}, x_{2}\right)= & \frac{m_{\mathrm{Z}}}{M}\left[2 \xi_{3}\left(x_{1}, x_{2}\right)\right], \\
g_{22}\left(x_{1}, x_{2}\right)= & \frac{m_{\mathrm{Z}}}{M}\left[2 \xi_{4}\left(x_{1}, x_{2}\right)\right],
\end{aligned}
$$

and

$$
\begin{aligned}
\xi_{1}\left(x_{1}, x_{2}\right)= & \frac{\mu_{0}}{2 \pi}\left\{-\frac{\left[e_{M}^{2}-\left(c_{M}-a_{1} x_{1}+x_{2}\right)^{2}\right]}{\left[\left(c_{M}-a_{1} x_{1}+x_{2}\right)^{2}+e_{M}^{2}\right]^{2}},\right. \\
& +\frac{\left[e_{M}^{2}-\left(-c_{M}-a_{1} x_{1}+x_{2}\right)^{2}\right]}{\left.\left[\left(-c_{M}-a_{1} x_{1}+x_{2}\right)^{2}+e_{M}^{2}\right]^{2}\right\}} \\
\xi_{2}\left(x_{1}, x_{2}\right)= & \frac{\mu_{0}\left\{-\frac{\left[e_{M}^{2}-\left(c_{M}+a_{2} x_{1}+x_{2}\right)^{2}\right]}{2 \pi}\left\{-\left[\left(c_{M}+a_{2} x_{1}+x_{2}\right)^{2}+e_{M}^{2}\right]^{2}\right.\right.}{}, \\
& +\frac{\left[e_{M}^{2}-\left(-c_{M}+a_{2} x_{1}+x_{2}\right)^{2}\right]}{\left.\left[\left(-c_{M}+a_{2} x_{1}+x_{2}\right)^{2}+e_{M}^{2}\right]^{2}\right\}} \\
\xi_{3}\left(x_{1}, x_{2}\right)= & \frac{\mu_{0} N}{2 \pi}\left\{\frac{-\left(d-a_{1} x_{1}+x_{2}\right)^{2}}{\left[\left(d-a_{1} x_{1}+x_{2}\right)^{2}\right]^{2}}+\frac{-\left(-d-a_{1} x_{1}+x_{2}\right)^{2}}{\left[\left(-d-a_{1} x_{1}+x_{2}\right)^{2}\right]^{2}}\right\}, \\
\xi_{4}\left(x_{1}, x_{2}\right)= & \frac{\mu_{0} N}{2 \pi}\left\{\frac{-\left(d+a_{2} x_{1}+x_{2}\right)^{2}}{\left[\left(d+a_{2} x_{1}+x_{2}\right)^{2}\right]^{2}},\right. \\
& \left.+\frac{-\left(-d+a_{2} x_{1}+x_{2}\right)^{2}}{\left[\left(-d+a_{2} x_{1}+x_{2}\right)^{2}\right]^{2}}\right\}
\end{aligned}
$$

where $a_{1}, a_{2}, b_{1}, b_{2}, c_{\mathrm{M}}, d, e_{\mathrm{M}}$ and $N$ are dimensional parameters. $\mu_{0}$, the permeability of free space, is a constant and equal to $4 \pi \times 10^{-7} \mathrm{H} / \mathrm{m}$.

\subsection{Electrical dynamics}

The electrical dynamics of the maglev suspension system can be treated as two independent inductance-resistance circuits as: 
$\dot{I}_{1}=-\frac{R_{1}}{L_{1}} I_{1}+\frac{K_{\mathrm{Al}}}{L_{1}} \dot{u}_{1}=\tau_{\mathrm{el}} I_{1}+K_{\mathrm{el}} u_{1}$,

$\dot{I}_{2}=-\frac{R_{2}}{L_{2}} I_{2}+\frac{K_{\mathrm{A} 2}}{L_{2}} U_{2}=\tau_{\mathrm{e} 2} I_{2}+K_{\mathrm{e} 2} u_{2}$,

where $R_{1}, R_{2}$ are the resistors of the electromagnet coils, $L_{1}$, $L_{2}$ are the inductors of the electromagnet coils, and $K_{\mathrm{A} 1}$, $K_{\mathrm{A} 2}$ are gains of the linear power amplifiers. $u_{1}, u_{2}$ are the control voltages to the amplifiers. They serves as the control input variables. The complete model then requires the combination of the mechanical and the electrical dynamics.

\section{Controller design}

The controller is proposed that will stabilize the system outputs, lateral translation $(X)$ and rotation with respect to $Z$ axis $(\theta)$, by two inputs, a control signal for inner tracks' stabilizers $\left(u_{1}\right)$ and the outer tracks' stabilizers $\left(u_{2}\right)$. The experimental maglev system composes of 5 induction-type proximity sensors to measure distances required to determine the control parameters (figure 3).

The range of the sensors are $0 \sim 2 \mathrm{~mm}$. The output of the sensor can be adjusted between 0 2 Volts, and the resolution of the sensor is $0.1 \%$ of the measurement range up to a frequency bandwidth of $\mathrm{DC}-3.3 \mathrm{kHz}(-3 \mathrm{~dB})$. In this experiment, the resolution is $2 \mu \mathrm{m}$. Using the Eular angle description, the relationship between a fixed coordinate $\mathrm{X}-\mathrm{Y}-\mathrm{Z}$ and the body coordinate $x-y-z$ can be written as

$\left[\begin{array}{l}X \\ Y \\ Z\end{array}\right]=\mathbf{T} \cdot\left[\begin{array}{l}x \\ y \\ z\end{array}\right]$

where

$$
\left.\begin{array}{rl}
\mathbf{T}=\left[\begin{array}{cc}
\cos \theta \cos \phi & -\sin \theta \cos \zeta+\cos \theta \sin \phi \sin \zeta \\
\sin \theta \cos \phi & \cos \theta \cos \zeta+\sin \theta \sin \phi \sin \zeta \\
-\sin \phi & \cos \phi \sin \zeta
\end{array}\right. \\
-\sin \theta \sin \zeta+\cos \theta \sin \phi \cos \zeta \\
-\cos \theta \sin \zeta+\sin \theta \sin \phi \cos \zeta \\
\cos \phi \cos \zeta
\end{array}\right] .
$$

is the transformation matrix. If the position of the sensors on the stage are

$$
s_{1, \mathrm{xyz}}=\left[\begin{array}{c}
s_{1, \mathrm{x}} \\
s_{1, \mathrm{y}} \\
s_{1, \mathrm{z}}
\end{array}\right]=\left[\begin{array}{c}
a_{\mathrm{s}} \\
b_{\mathrm{s}} \\
0
\end{array}\right] \quad, \quad s_{2, \mathrm{xyz}}=\left[\begin{array}{c}
s_{2, \mathrm{x}} \\
s_{2, \mathrm{y}} \\
s_{2, \mathrm{z}}
\end{array}\right]=\left[\begin{array}{c}
a_{\mathrm{s}} \\
0 \\
0
\end{array}\right]
$$

$$
\begin{aligned}
& s_{3, x y z}=\left[\begin{array}{l}
s_{3, x} \\
s_{3, y} \\
s_{3, z}
\end{array}\right]=\left[\begin{array}{c}
a_{s} \\
-b_{s} \\
0
\end{array}\right], \quad s_{4, x y z}=\left[\begin{array}{c}
s_{4, x} \\
s_{4, y} \\
s_{4, z}
\end{array}\right]=\left[\begin{array}{c}
-a_{s} \\
b_{s} \\
0
\end{array}\right], \\
& s_{s, x y z}=\left[\begin{array}{l}
s_{5, x} \\
s_{5, y} \\
s_{s, z}
\end{array}\right]=\left[\begin{array}{c}
-a_{s} \\
-b_{s} \\
0
\end{array}\right]
\end{aligned}
$$

where $a_{\mathrm{s}}, b_{\mathrm{s}}$ are known dimensions. If one assume small pitch and yaw angle for the precision servo, the sensor position can be calculated as

$$
\begin{aligned}
& s_{1, \mathrm{XYZ}}=\left[\begin{array}{l}
s_{1, \mathrm{X}} \\
s_{1, \mathrm{Y}} \\
s_{1, \mathrm{Z}}
\end{array}\right]=\left[\begin{array}{c}
a_{\mathrm{s}}-b_{\mathrm{s}} \theta+X \\
a_{\mathrm{s}} \theta+b_{\mathrm{s}} \\
-a_{\mathrm{s}} \phi+b_{\mathrm{s}} \zeta+Z
\end{array}\right], \\
& s_{2, \mathrm{XYZ}}=\left[\begin{array}{l}
s_{2, \mathrm{X}} \\
s_{2, \mathrm{Y}} \\
s_{2, \mathrm{Z}}
\end{array}\right]=\left[\begin{array}{c}
a_{\mathrm{s}}+X \\
a_{\mathrm{s}} \theta \\
-a_{\mathrm{s}} \phi+Z
\end{array}\right], \\
& s_{3, \mathrm{XYZ}}=\left[\begin{array}{l}
s_{3, \mathrm{X}} \\
s_{3, \mathrm{Y}} \\
s_{3, \mathrm{Z}}
\end{array}\right]=\left[\begin{array}{c}
a_{\mathrm{s}}+b_{\mathrm{s}} \theta+X \\
a_{\mathrm{s}} \theta-b_{\mathrm{s}} \\
-a_{\mathrm{s}} \phi-b_{\mathrm{s}} \zeta+Z
\end{array}\right], \\
& s_{4, \mathrm{XYZ}}=\left[\begin{array}{l}
s_{4, \mathrm{X}} \\
s_{4, \mathrm{Y}} \\
s_{4, \mathrm{Z}}
\end{array}\right]=\left[\begin{array}{c}
-a_{\mathrm{s}}-b_{\mathrm{s}} \theta+X \\
-a_{\mathrm{s}} \theta+b_{\mathrm{s}} \\
a_{\mathrm{s}} \phi+b_{\mathrm{s}} \zeta+Z
\end{array}\right], \\
& s_{\mathrm{s}, \mathrm{XYZ}}=\left[\begin{array}{l}
s_{5, \mathrm{X}} \\
s_{5, \mathrm{Y}} \\
s_{5, \mathrm{Z}}
\end{array}\right]=\left[\begin{array}{c}
-a_{\mathrm{s}}+b_{\mathrm{s}} \theta+X \\
-a_{\mathrm{s}} \theta-b_{\mathrm{s}} \\
a_{\mathrm{s}} \phi-b_{\mathrm{s}} \zeta+Z
\end{array}\right] .
\end{aligned}
$$

The attitude of the stage can now be calculated by the five sensor measurements.

$$
\mathbf{H}=\left[\begin{array}{l}
h_{1}(\theta, \phi, \zeta, X, Z) \\
h_{2}(\theta, \phi, \zeta, X, Z) \\
h_{3}(\theta, \phi, \zeta, X, Z) \\
h_{4}(\theta, \phi, \zeta, X, Z) \\
h_{5}(\theta, \phi, \zeta, X, Z)
\end{array}\right]=\left[\begin{array}{c}
s_{1, X}-a_{s} \\
s_{2, Z} \\
s_{3, \mathrm{X}}-a_{\mathrm{s}} \\
s_{4, Z} \\
s_{s, Z}
\end{array}\right]=\left[\begin{array}{c}
-b_{s} \theta+X \\
-a_{s} \phi+Z \\
b_{s} \theta+X \\
a_{s} \phi+b_{s} \zeta+Z \\
a_{\mathrm{s}} \phi-b_{\mathrm{s}} \zeta+Z
\end{array}\right]
$$

The carriage magnets in the system receive the forces from the magnetic tracks. There are four carriage magnets in figure 2 located among the centerline of the magnetic tracks. It is nature to decompose the input-output pairs by pairing the force-receiving magnet with the closet sensor. The five sensors thus derived five subsystems. As will be discussed later, this configuration resulted in a system that is very difficult to tune.

An alternate configuration thus arises from consideration the system kinematics. The electric wires to the two inside 
rails are connected in series, thus they generate a force that lift up the front end of the carriage (figure 2). Likewise, the outside rails generate a force that lift up the rear end of the carriage. Two pair of the side forces can be treated similarly to move the front and rear end of the carriage in the $x$ direction. The difference in the two pairs of side forces generates a torque that controls the yaw motion.

A hybrid controller that combines the bang-bang control for near saturation control in conjunction with a high performance pole-placement controller then serves as the servo controller. The controller parameters are tuned by assuming a linear system for experimental system identification. Notice that the system model is experimentally determined, therefore the control parameter should achieve the desired control if the subsystem configuration is reasonable.

\section{Experimental Setup}

The maglev suspension system considered is an electrically and mechanically integrated system (figure 5). The maglev suspension system consists of the mechanism, a set of power amplifiers as actuators, a Pentium $233 \mathrm{PC}$ as the controller, inductive gauging sensors as feedback sources, a 12-bit ADC and a 12-bit $\mathrm{DAC}$ as system input-output devices. The resolution of sensors is $4 \mu \mathrm{m}$ and the bandwidth of sensors is $3.3 \mathrm{kHz}$. The control inputs are limited within \pm 9 Volts to protect the stabilization coils. Due to the computational power of Pentium 233 PC, high-speed sampling is possible when complex control algorithm is implemented and because the calculation for the controller is very straight, the sampling frequency for the five axes can be as high as $2.5 \mathrm{KHz}$.

\section{Experimental results and discussions}

The goals set for the maglev suspension system are a demonstration of stability, decoupling of the degrees-of-freedom, improving the system's rigidity. The result from the proposed first partition is shown in figures 6 and 7. Due to the space limit, only the more critical responses are included. Figure 6 shows the computed closed-loop frequency response from the experimentally identified subsystem from exciting the first pair of literal stabilizing coil to the first sensor output. The closed-loop bandwidth is $66 \mathrm{~Hz}$. Figure 6 shows the step response of the same subsystem. Very good performance is observed.

Figures 8 and 9 shows the frequency and step responses for the sensor 3 subsystem. The step response looks poor and the closed-loop bandwidth is calculated to be $2.7 \mathrm{~Hz}$. Both subsystem for sensor 3 and 4 shows particular low bandwidth. Increasing the loop gain by adjusting the controller parameters resulted in oscillatory responses. This is a result of improper subsystem partition. The system is stable when separately tuned, but when all the subsystem loops are activated, the side forces generated by the sensor 3 subsystem input coil also generate strong coupling effect to the other subsystems. Therefore, increasing loop affect the stabilizing action in the other control loops.

As an attempt to correct this effect, the new subsystem partitioning is carried out. Figure 10 shows the closed-loop frequency response for the literal motion, and figure 11 shows the step response to the same subsystem.

The rest of the subsystems achieve similar performances. The bandwidths for the subsystems when all the loops are activated are consistently $53.15 \mathrm{~Hz}, 50.93 \mathrm{~Hz}, 46.95 \mathrm{~Hz}$, $53.63 \mathrm{~Hz}$, and $41.38 \mathrm{~Hz}$. We see that the coupling effect is successfully suppressed and the overall system perform in consistent with the separate subsystem.

\section{Conclusions}

In this paper, a mechatronics approach to simplify the servo design for the maglev system is proposed. The mechatronics approach basically divided the complicated system into multiple local loops that may be separately controlled. The break up of the system enables the use of simple SISO control loops for the subsystems. This also lifted the limitation on the sampling rate derived from the complex controller computation. However, this paper also pointed out that careless assignment of the sensor/actuator pairs could lead to very hard to tune overall systems. The worse case can even cause system instability. A kinematics analysis provided in the paper leads to reasonable subsystem partitions, and overall system behavior that is consistent with the separate subsystem performance is achieved.

\section{Acknowledgements}

This work is supported by the National Science Council, 
Taiwan R.O.C. under project No. NSC88 -2213 -E -002 $-083$.

\section{Reference}

[1]. Sinha, P. K., Electromagnetic Suspension, "Dynamics and Control". London. Peter Peregrinus, 1987.

[2]. Cho, D., Y. Kato, and D. Spilman, "Sliding Mode and Classical Control of Magnetic Levitation Systems". IEEE Control Syst. Mag., vol. 13,no. 1, pp. 42-48, 1993.

[3]. Atherton, D. L., "Maglev using paermagnets", IEEE Trans. Magn., vol. 16,no. 1., pp. 146-148, 1980.

[4]. Morishita, M., T. Azukizawa, S. Kanda, N. Tamura, and T. Yokoyama, "A new maglev system for magnetically levitated carrier system", 1986 Int. Conf. Maglev and Linear Drives, Vancouver, B. C., May 1986.

[5]. Tsukamoto, O., K. Yasuda, and J. Z. Chen, " A New Magnetic Levitation System with ac Magnets", IEEE Trans. Magn., vol. 24, no. 2, pp. 1497-1500, 1988.

[6]. Williams, R., J. R. Matey, Y. Arie, and J. Rathee, "The effect of mass and pole strength on the levitation height of the magnet over a superconductor", J. App. Phys., vol. 65, no. 9, pp. 3583-3585, 1989.

[7]. Wu, K. N., and L. L. Chen, "Adaptive control of a four-track maglev system", Journal of Control Systems and Technology 4, 4,pp. 295-302, 1996.

[8]. Wang, I.Y., A Magnetic Levitation Silicon Wafer Transport System. Ph.D. Thesis, The University of Texas at Austin, 1993.

[9]. Huang, Chao-Ming, Jia-Yush Yen, Min-Shin Chen, "Adaptive nonlinear control of repulsive maglev suspension systems," Control Engineering Practice, To appear Sep issue (2000).

[10]. Tsukamoto, O., Yasuda, K. \& Chen, J. Z. (1988). A new magnetic levitation system with AC magnets. IEEE Transactions on Magnetics, 24, (2), 1497-1500.

[11]. Williams, R., Matey, J. R., Arie, Y. \& Rathee, J. (1989). The effect of mass and pole strength on the levitation height of the magnet over a superconductor. Journal of Applied Physics, 65, (9), 3583-3585.

[12]. Wu, K. N. \& Fu, L. C. (1996). Adaptive control of a four-track maglev system, Journal of Control Systems and Technology, 4, (4), 295-302.

[13]. Huang, C. M. (1999). Nonlinear Controller Design of Repulsive Maglev Suspension Guiding System. $\mathrm{PhD}$ thesis, The National Taiwan University.

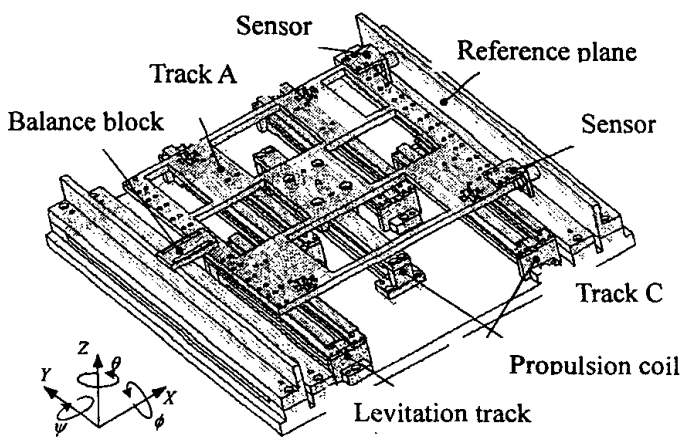

Figure 3. A 3-D view of the maglev suspension system

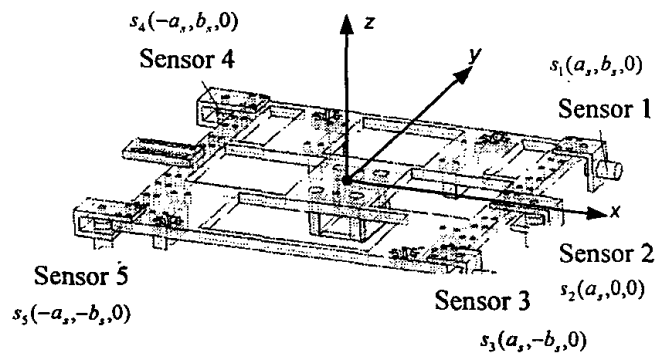

Figure 4 Sensor configurations for the maglev system

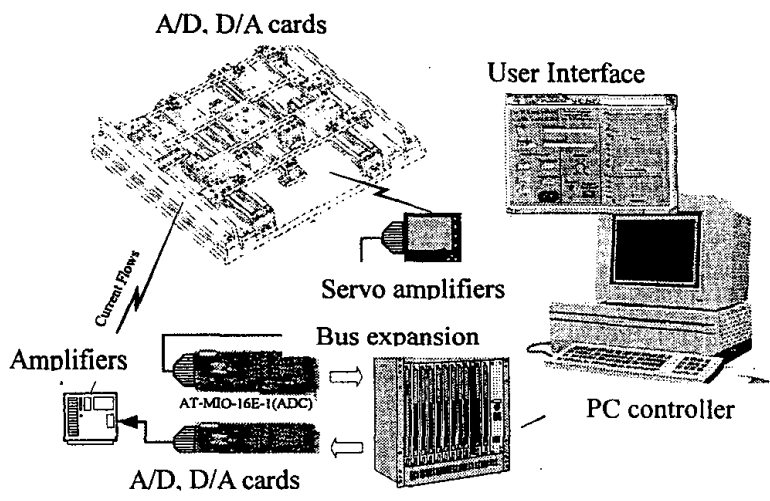

Fig. 5. The configuration of the repulsive maglev suspension system 


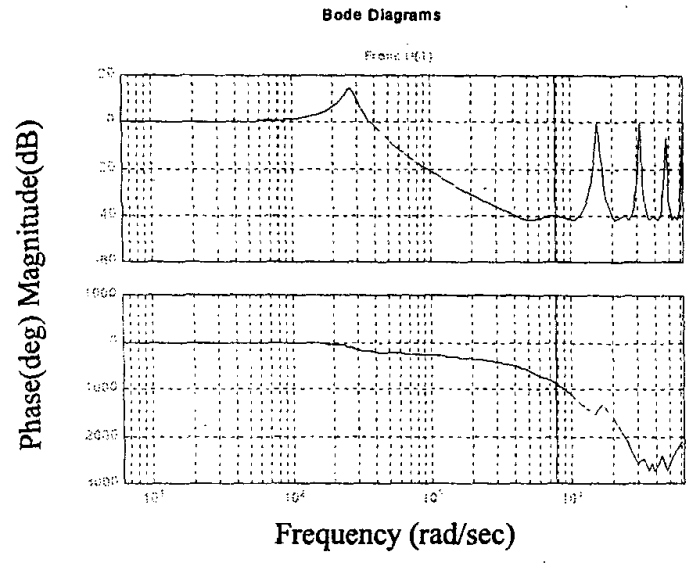

Figure 6 Frequency response tor the sensor 1 subsystem

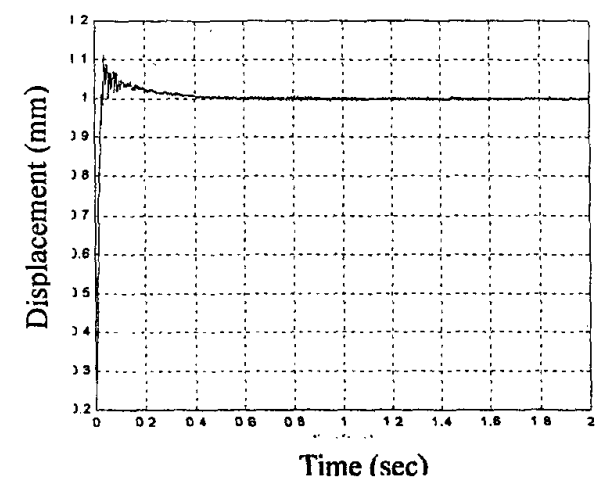

Figure 7 Step response for the sensor 1 subsystem Bode Diagrams

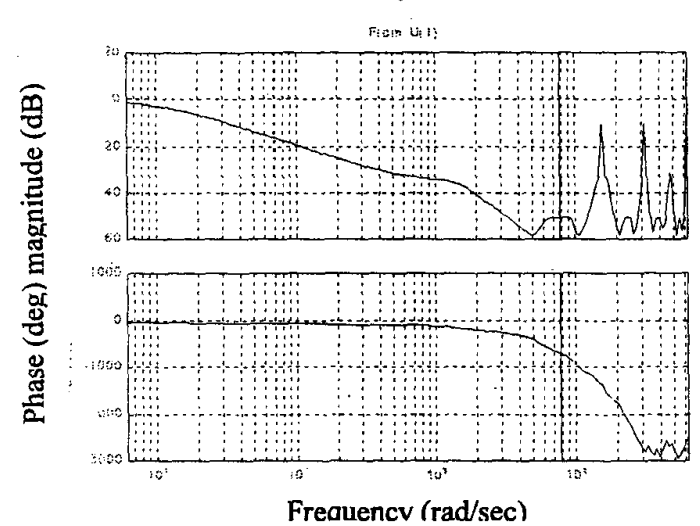

Figure 8 Frequency response for the sensor 3 subsystem

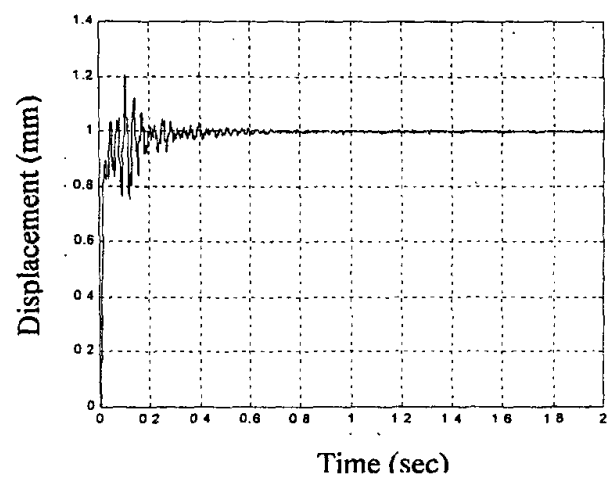

Figure 9. Step response for the sensor 3 subsystem

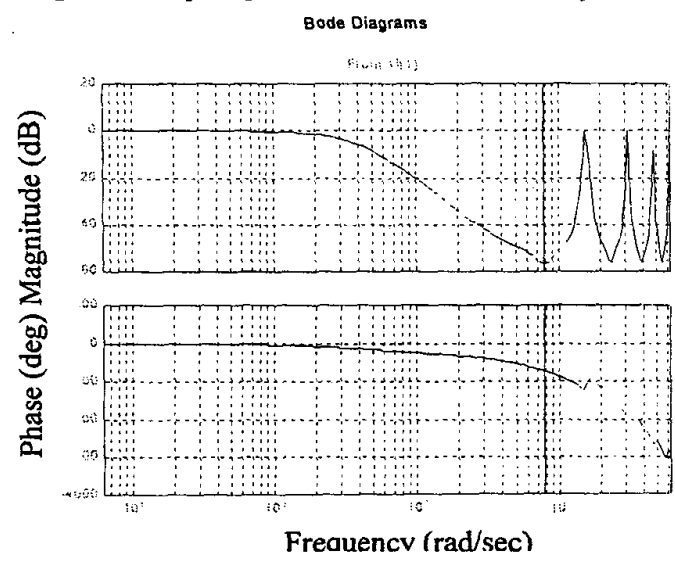

Figure 10 Literal subsystem frequency response

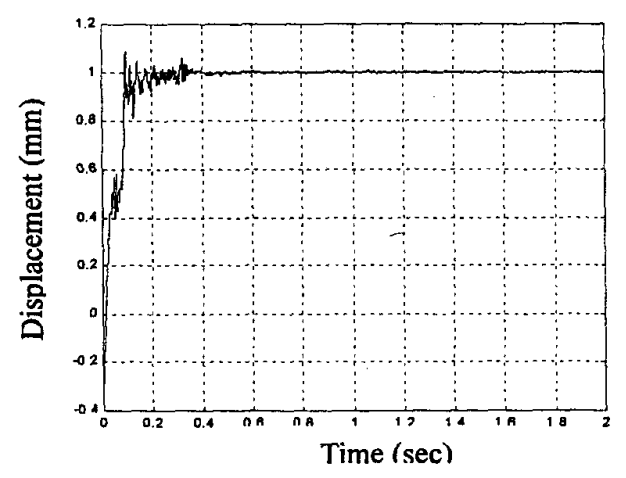

Figure 11 Literal subsystem step response 